\title{
. \\ Hemolysis contributes to anemia during long-duration space flight
}

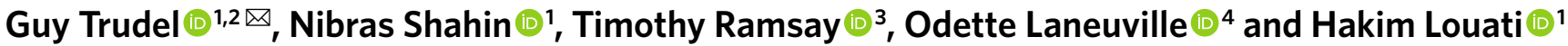

\begin{abstract}
Anemia in astronauts has been noted since the first space missions, but the mechanisms contributing to anemia in space flight have remained unclear. Here, we show that space flight is associated with persistently increased levels of products of hemoglobin degradation, carbon monoxide in alveolar air and iron in serum, in 14 astronauts throughout their 6-month missions onboard the International Space Station. One year after landing, erythrocytic effects persisted, including increased levels of hemolysis, reticulocytosis and hemoglobin. These findings suggest that the destruction of red blood cells, termed hemolysis, is a primary effect of microgravity in space flight and support the hypothesis that the anemia associated with space flight is a hemolytic condition that should be considered in the screening and monitoring of both astronauts and space tourists.
\end{abstract}

As humankind plans extraterrestrial travel, understanding the health implications of living in space will be critical to planning safe journeys. Space anemia was previously documented and characterized by a $10-12 \%$ decrease in red blood cell (RBC) mass happening in the first 10 days in space ${ }^{1}$. Current understanding of space anemia is that the decrease in RBCs constitutes an acute adaptation to major hemodynamic events of cephalad fluid shifts, hemoconcentration and low erythropoietin (EPO) levels upon entering microgravity ${ }^{1,2}$. Thereafter, beyond 10 days in space, when the hemoglobin concentration returns to near-earthly values, erythrocytic regulation would proceed normally, but this has not been measured precisely ${ }^{2}$. Recently, astronauts were found to remain mildly hemoconcentrated throughout long-duration mission ${ }^{3}$, and epidemiological data showed that the severity, time to recovery and longitudinal effects of postflight anemia were proportional to the time spent in space ${ }^{4}$. These reports challenged the current understanding of space anemia. Longer missions to the moon and Mars, as well as space tourism and commercialization, require a better understanding of space-induced anemia. Because astronaut orthostatism, exercise tolerance and fatigue are key functions affected by anemia, RBC management will be vital for human missions landing on extraterrestrial worlds without medical supervision.

While a variety of hypothetical causes (e.g., RBC dysfunction, decreased production, sequestration or increased destruction) have been proposed for space anemia, the physiologic mechanisms are not fully established ${ }^{5}$, and studying these mechanisms in space is challenging. Hemolysis releases hemoglobin, and heme rings are broken down by heme oxygenases ${ }^{6}$. Each heme molecule produces one ferrous iron, one carbon monoxide ( $\mathrm{CO}$ ) and one biliverdin molecule. In basal conditions, approximately $85 \%$ of endogenously produced $\mathrm{CO}$ arises from hemoglobin ${ }^{6}$. The quantification of CO molecules eliminated is therefore a direct measure of hemolysis. Recently developed methods to precisely quantify endogenous $\mathrm{CO}$ now permit the measurement of hemolysis in space ${ }^{7}$. Using these methods, 20 participants showed increased hemolysis (by an average of $23 \%$ ) throughout 60 days of the antiorthostatic bed-rest microgravity analogue ${ }^{8}$. These findings suggested that increased hemolysis may be an important primary effect of the microgravity analogue, a hypothesis never tested in space. We therefore measured hemolysis markers in breath and blood samples from astronauts preflight, four times inflight and up to 1 year after their 6-month missions to the International Space Station (ISS).

A total of 14 astronauts were recruited (11 men and 3 women; $45 \pm 7$ years) between 2015 and 2020 (Fig. 1a). The astronauts flew ISS missions of $167 \pm 31$ days duration. Each astronaut collected alveolar and ambient air samples as well as blood samples according to a prespecified schedule (Fig. 1b,c). The space samples were downmassed using automated reentry vehicles.

Figure 2a shows that mean baseline CO elimination was 1,662 ppb (95\% confidence interval (CI), 1,433-1,891). After an average of $5 \pm 1$ days on the ISS, CO elimination was 2,627 ppb $(95 \%$ CI, 2,342-2,913); the mean increase from preflight was $56 \%(95 \%$ CI, 36-76; Supplementary Table 1). Increased hemolysis may have started earlier than day 5 (the first sampling time). The finding of increased CO elimination was corroborated by the observation that serum iron and iron carrier proteins transferrin and ferritin also increased (Fig. 2b-d). Large elevations in heme degradation products both in alveolar air and blood samples constitute the first direct demonstration of upregulated hemolysis in space and support the hypothesis that space-related anemia is a hemolytic condition.

The next question was whether the hemolysis stopped when the hemoglobin concentration reached near-earthly values after 10 days in space. CO elimination in space over 6 months (average of 46 measures) was increased $54 \%$ (95\% CI, 39-70) compared to preflight (Fig. 2a; Supplementary Tables 1 and 2). Figure 2b-d shows that serum iron, transferrin saturation and ferritin levels in space (average of 55 measures each) were also higher compared to preflight throughout the 6-month missions (Supplementary Tables 1 and 2). Persistently increased CO elimination in space showed that hemolysis was not an acute adaptation to hemodynamic alterations upon entering microgravity. Increased hemolysis rather constituted a primary effect of exposure to space. This conclusion is strengthened by the postflight data. The astronaut first postflight measure (taken 4 days after landing, separated by only 14 days from the last inflight measure) showed sharp decreases in hemolysis markers coinciding with the return to Earth's surface gravity (Fig. 2a-d). The pattern of hemolysis in the three phases of the study (on Earth, in

'Bone and Joint Research Laboratory, Ottawa Hospital Research Institute, Ottawa, Ontario, Canada. ${ }^{2}$ Division of Physical Medicine and Rehabilitation, Department of Medicine and Department of Biochemistry, Microbiology and Immunology, Faculty of Medicine, University of Ottawa, Ottawa, Ontario, Canada. ${ }^{3}$ School of Epidemiology and Public Health, University of Ottawa, Ottawa, Ontario, Canada. ${ }^{4}$ Department of Biology, Faculty of Science, University of Ottawa, Ottawa, Onatrio, Canada. 凶e-mail: gtrudel@toh.ca 
a

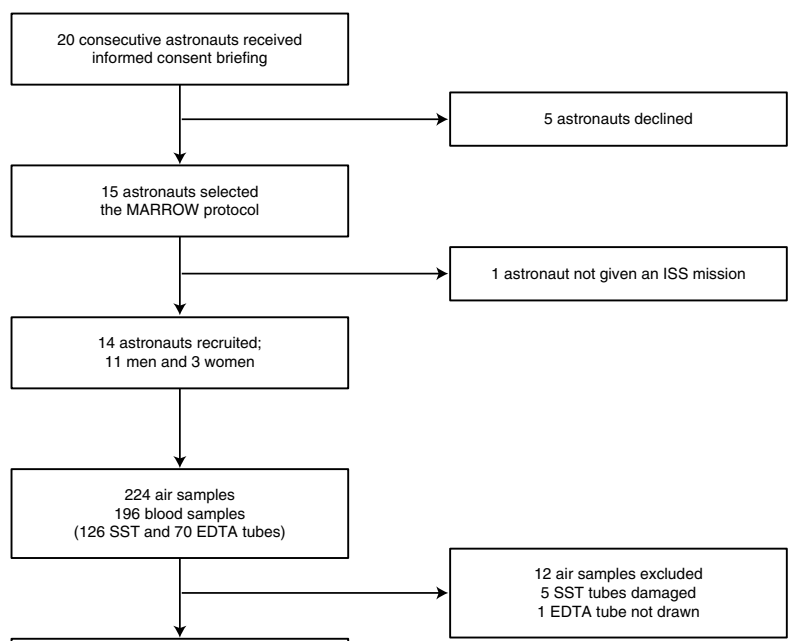

212 measures of $\mathrm{CO}$ in air samples
$121-125$ measures of various analytes

21-125 measures of various analytes from SSTs 69 measures of

b
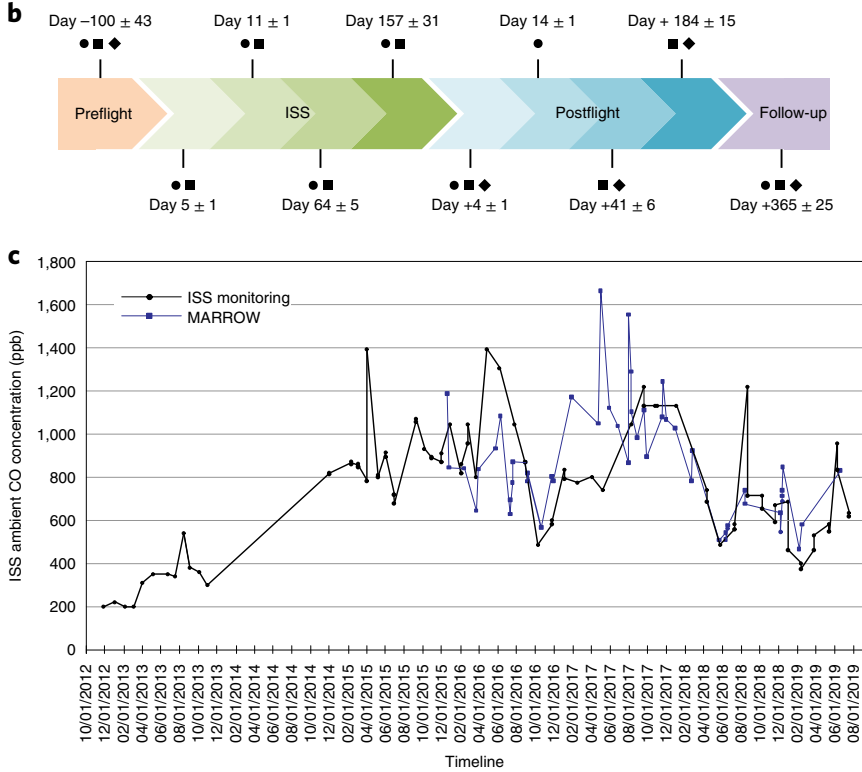

Fig. 1 | Bone Marrow Adipose Reaction: Red Or White (MARROW) study design and outcomes. a, Flow chart showing recruitment and samples harvested. SST, serum separator tube. $\mathbf{b}$, Schematic showing the study design and planned sample collection. Astronauts recruited to the MARROW study performed serial measures at prespecified time points preflight, onboard the ISS and up to 1 year postflight. The average number of days ( \pm s.d.) of sampling in relation to takeoff and landing are indicated. The interval between the last $\mathrm{CO}$ sampling in space and the first sampling on Earth after landing was $14 \pm 5$ days. Circles, CO measurements; squares, serum analytes (iron, transferrin percent saturation, ferritin, haptoglobin, bilirubin, C-reactive protein and EPO); diamonds, complete blood counts (CBCs) and reticulocytes. $\mathbf{c}$, Time course of ambient air $\mathrm{CO}$ concentration onboard the ISS.

space and back on Earth) confirmed that increased hemolysis was tightly linked to the space environment.

Increased hemolysis as a primary effect of exposure to space constitutes a paradigm shift in our understanding of space ane$\mathrm{mia}^{5}$. Major pathophysiological implications include that space anemia may be dependent on the duration of exposure to space and independent of fluid shifts, EPO levels and the RBC production environment on Earth versus in space and that it may trigger a compensatory increase in $\mathrm{RBC}$ production and enhance nutritional needs. Persistent hemolysis during space missions suggests that the longer the exposure, the worse the anemia. A similar conclusion was reached after analyzing data from 711 missions from American and Canadian astronauts between 1968 and 2015 (ref. ${ }^{4}$ ). Increased hemolysis was measured both during fluid shifts (inflight days 5 and 11) and long after fluid shifts were completed (inflight days 64 and 157), dissociating it from a causation effect. Increased hemolysis appeared EPO independent. In the current trial, increased hemolysis was sustained under both suppressed (inflight days 5 and 11) and corrected EPO levels (inflight days 64 and 157; Fig. 2e). In the bone marrow, colony-forming unit erythroid cells depend on EPO for survival and differentiation into erythroblasts, with only the latter synthesizing hemoglobin ${ }^{9}$. Reticulocytes and mature RBCs lack $\mathrm{EPO}$ receptors and cease responding to $\mathrm{EPO}^{9}$. Colony-forming unit erythroid cell suppression under low-EPO conditions should not enhance heme degradation, whereas degradation of erythroblasts, reticulocytes and mature RBCs would. Therefore, our data support increased hemolysis of EPO-independent erythroblasts and/or more differentiated RBCs. Upon entering space, all astronaut RBCs are terrestrial born. Based on normative RBC lifespan, after 120 days in space, all RBCs are space born. In this study, both RBC populations hemolyzed at higher rates while in space. At postflight day 4, the hemolysis of space-born RBCs sharply decreased. These results suggest that intrinsic characteristics of Earth-born or space-born RBCs do not influence hemolysis. Space hemolysis attenuates the initial hemoconcentration caused by fluid shifts obviating the need for compensatory RBC production. Later in the mission, a continuing $54 \%$ increased hemolysis risks causing severe anemia. That astronauts remain mildly hemoconcentrated throughout long-duration space missions ${ }^{3}$ implies a compensatory increased RBC production. Enhanced RBC production in space was not directly measured in this study, but robust EPO levels at inflight days 64 and 157 and increased reticulocytes at postflight day 4 supported stimulated RBC production (Fig. 2e,f and Supplementary Table 2). Hemolysis with compensatory RBC production will expand bone marrow erythropoietic activity, the higher energy requirements of which must be factored in astronaut nutritional planning. C-reactive protein levels remained unchanged throughout ISS missions, arguing against an inflammatory component to space anemia from background inflammation in space or free hemoglobin as a potent inflammatory inducer (Supplementary Tables 1-3).

The return to Earth's surface also causes major hemodynamic changes, as fluid shift reversal increases plasma volume and dilutes $\mathrm{RBCs}^{1,5}$. Our astronaut cohort had an $8.8 \%$ (95\% CI, 8.1-9.5) decrease in hemoglobin concentration 4 days after landing compared to preflight (Fig. $2 \mathrm{~g}$ and Supplementary Table 2) with 5 of 13 astronauts (38.5\%) reaching clinical levels of anemia ${ }^{10}$. Low hemoglobin concentrations postflight indicate that astronauts' first landing measure, just like inflight measures past 10 days in space, originated from fewer $\mathrm{RBCs}^{1}$. These predicted proportionately lower CO elimination, iron and iron carrier protein concentrations. The implication is that the hemolysis figures we report compared to preflight data, as high as they are, are likely underestimating the actual rate of hemolysis at these time points.

The next question was how reversible these effects were up to 1 year after long-duration missions. Erythrocytic effects of space include increased hemolysis (herein reported), correspondingly elevated RBC production that is assumed and previously reported hemoglobin reset at higher concentrations ${ }^{3}$. Previous spaceflight data suggested persistent erythrocytic effects with higher hemoglobin concentrations $\left(0.27 \mathrm{~g} \mathrm{dl}^{-1}\right)$ in 58 astronauts 1 year after missions averaging 145 days $^{4}$. In the current trial, 1 year after landing, CO elimination was 30\% (95\% CI, 2-57) higher, reticulocytes were $16 \%$ (95\% CI, 7-24) higher and hemoglobin concentration 

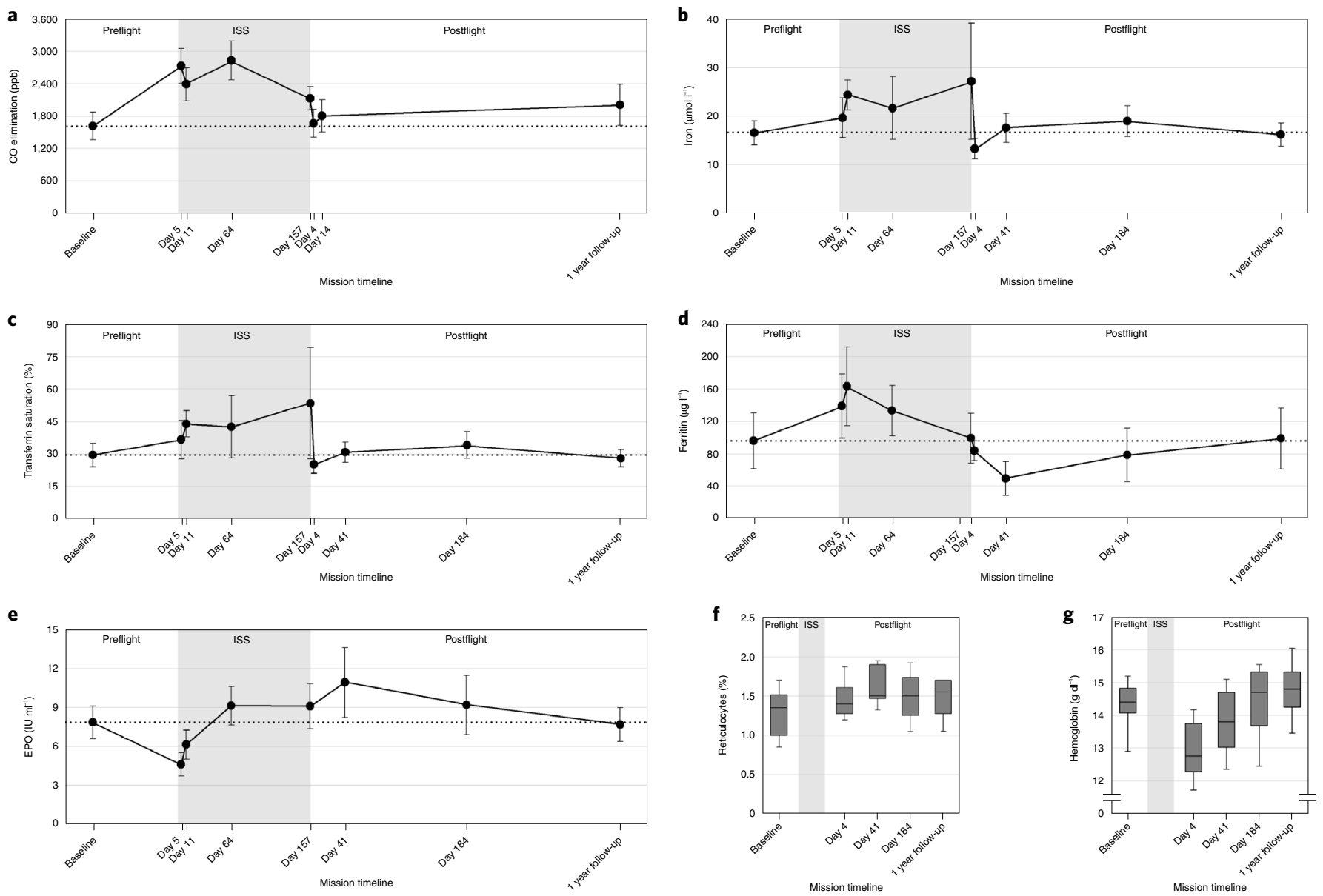

Fig. 2 | Hemolysis and erythropoietic markers of astronauts preflight, onboard the ISS and postflight. a, CO elimination. b-e, Serum iron concentrations (b), transferrin saturation (c), ferritin concentrations (d) and EPO concentrations (e). Data points and error bars represent mean values with $\pm 95 \% \mathrm{Cls}$. Shaded areas represent data from ISS samples. $\mathbf{f}, \mathbf{g}$, Percentage of reticulocytes $(\mathbf{f})$ and hemoglobin concentrations $(\mathbf{g})$. The center line in the box plots represents the median, the upper limit of the box plots represents the third quartile (75th percentile), the lower limit of the box plots represents the first quartile (25th percentile), the upper whisker is the maximum value of the data that are within 1.5 times the interquartile range over the 75th percentile and the lower whisker is the minimum value of the data that are within 1.5 times the interquartile range under the 25 th percentile. $N=14$ astronauts who provided individual samples at five, eight or nine independent time points according to the outcome.

was 3.5\% (95\% CI, 1.5-5.4) (0.50 g dl-1) higher than preflight levels (Fig. 2a,f,g and Supplementary Tables 1 and 2). These data support increased hemolysis, RBC production and hemoglobin concentration 1 year after long-duration space exposure. These erythrocytic space effects may not be permanent, as longitudinal astronaut data over 20 years predicted lower lifelong hemoglobin concentrations $\left(-0.001\right.$ and $-0.002 \mathrm{~g} \mathrm{dl}^{-1}$ for male and female astronauts, respectively) for every day in microgravity. Erythrocytic effects 1 year postflight support long-term monitoring and consideration on the optimal interval between repeated space missions.

The anatomic site and mechanism of space hemolysis remain unknown. Hemolysis can be intravascular or extravascular in the spleen, liver or bone marrow. Increased heme degradation without haptoglobin depletion in this trial argues against intravascular hemolysis (Supplementary Tables 1 and 2). Erythroblast and reticulocyte destruction in the bone marrow before their egress into the circulation (sometimes called inefficient erythropoiesis) could arise from marrow adipose tissue accumulation from the lack of bone stimulation in space and dyserythropoiesis ${ }^{11}$. Altered size and shape of erythrocytes or the spleen in space may increase extravascular hemolysis ${ }^{12}$. Mature RBC lifespan may be shortened by mitochondrial stress and dysregulation ${ }^{13}$. Gene modifiers (e.g., BCL11A and
KLF1) modulate the expression of globin genes ${ }^{14}$. The regulation of gene expression of these globin modifiers involves the methylation state of the DNA regions impacting their expression ${ }^{15}$. DNA hypomethylation in the $\beta$-globin gene cluster was linked to ineffective erythropoiesis in thalassemia ${ }^{15}$. Interestingly, altered methylation levels in CD4 and CD8 lymphocytes were reported after 1 year in space $^{2}$. Space or radiation effects on DNA methylation of regulatory regions controlling the expression of globin and gene modifiers constitute potential mechanisms of space hemolysis. Elucidating the site and mechanism of space hemolysis is crucial to developing successful mitigation strategies. The specific exercise and nutritional countermeasures of modern space travel did not prevent hemolysis and postflight anemia in our cohort, and both could have health consequences. Increased $\mathrm{CO}$ levels from space hemolysis can have second-messenger modulatory effects on intracellular processes of the cardiac, vascular, ocular, bone, nutrition, circadian cycle, orthostatic hypotension, brain and muscle systems of astronauts ${ }^{16,17}$.

Space tourism will considerably expand the number of space travelers ${ }^{18}$. Medical screening of future astronauts and space tourists might benefit from a preflight profiling of globin gene and modifiers. Postlanding monitoring should cover conditions affected by anemia and hemolysis. Monitoring individual astronaut's levels of 
hemolysis during mission may be indicated to reduce health risks ${ }^{18}$. Our results support adding space travel to the list of etiologies for hemolytic anemia.

Erythrokinetic response to microgravity may have critical relevance to understand erythroid physiology and pathophysiology in contexts other than space flight. Data from the mid-1990s focusing on the acute hemoconcentration entering space led to hypotheses on normal erythroid mass control, prevention from becoming excessive, hypoxic environments, polycythemia and EPO withdrawal ${ }^{5}$. Preferential hemolysis of younger erythrocytes after descent from altitude is currently debated ${ }^{19,20}$. Our findings of continuous hemolysis in space carry broad implications for populations on Earth with increased hemolysis or high prevalence of unexplained anemia, including patients receiving critical care, patients with chronic anemia, older patients, sedentary patients and bedridden patients ${ }^{21-23}$. Like astronauts, the environment of these individuals is characterized by a marked decrease in skeleton stimulation and decreased standing time. These individuals experience cardiovascular deconditioning, muscle atrophy and osteopenia. Potential alterations in bone marrow and spleen functions in space may be relevant to pathophysiological changes in their erythrocytic control. Our findings support investigating whether increased hemolysis contributes to anemia on Earth and could be targeted by specific physical rehabilitation strategies.

Limitations of this study include potential confounding factors associated with exposure to space, including fluid shifts, ambient $\mathrm{CO}$ concentration, altered circadian cycle and muscle atrophy. These factors were addressed with ambient CO sampling with each alveolar sample, chronostandardization and serial measures throughout ISS missions (Fig. 1c). Hemolysis with longer space exposure, such as 1-year ISS or Moon missions or journeys to Mars, and cumulative exposure constitute knowledge gaps. Recruitment was limited to astronauts, which threatens the generalization of these findings to space tourists. The unequal sex distribution precluded a robust examination of potential physiological differences (Supplementary Tables 3 and 4).

RBC regulation demonstrated major disruptions in space compared to on Earth. Increased hemolysis by $54 \%$ was a primary effect of exposure to space in astronauts that persisted throughout their long-duration missions and may constitute the leading mechanism of space-related anemia. Space hemolysis should be considered in the screening, monitoring and follow-up of astronauts launching on space exploration missions, as well as space tourists.

\section{Online content}

Any methods, additional references, Nature Research reporting summaries, source data, extended data, supplementary information, acknowledgements, peer review information; details of author contributions and competing interests; and statements of data and code availability are available at https://doi.org/10.1038/ s41591-021-01637-7.

Received: 27 May 2021; Accepted: 22 November 2021; Published online: 14 January 2022

\section{References}

1. Leach, C. S. \& Johnson, P. C. Influence of spaceflight on erythrokinetics in man. Science 225, 216-218 (1984).

2. Garrett-Bakelman, F. et al. The NASA Twins Study: a multidimensional analysis of a year-long human spaceflight. Science 364, eaau8650 (2019).
3. Kunz, H. et al. Alterations in hematologic indices during long-duration spaceflight. BMC Hematol. 17, 12 (2017).

4. Trudel, G., Shafer, J., Laneuville, O. \& Ramsay, T. Characterizing the effect of exposure to microgravity on anemia: more space is worse. Am. J. Hematol. 95, 267-273 (2020).

5. Alfrey, C. P. et al. Neocytolysis: physiological down-regulator of red-cell mass. Lancet 349, 1389-1390 (1997).

6. Abraham, N., Drummond, G., Lutton, J. \& Kappas, A. The biological significance and physiological role of heme oxygenase. Cell Physiol. Biochem. 6, 129-168 (1996).

7. Shahin, N., Louati, H. \& Trudel, G. Measuring human hemolysis clinically and in extreme environments using endogenous carbon monoxide elimination. Ann. Biomed. Eng. 48, 1540-1550 (2020).

8. Culliton, K., Louati, H., Laneuville, O., Ramsay, T. \& Trudel, G. Six degrees head-down tilt bed rest caused low-grade hemolysis: a prospective randomized clinical trial. NPJ Microgravity 7, 4 (2021).

9. Velten, L. et al. Human haematopoietic stem cell lineage commitment is a continuous process. Nat. Cell Biol. 19, 271-281 (2017).

10. World Health Organization. Haemoglobin Concentrations for the Diagnosis of Anaemia and Assessment of Severity (WHO, 2011).

11. Adler, B. J., Kaushansky, K. \& Rubin, C. T. Obesity-driven disruption of haematopoiesis and the bone marrow niche. Nat. Rev. Endocrinol. 10, 737-748 (2014).

12. Kristiansen, M. et al. Identification of the haemoglobin scavenger receptor. Nature 409, 198-201 (2001).

13. da Silveira, W. A. et al. Comprehensive multi-omics analysis reveals mitochondrial stress as a central biological hub for spaceflight impact. Cell 183, 1185-1201 (2020).

14. Giardine, B. et al. Updates of the HbVar database of human hemoglobin variants and thalassemia mutations. Nucleic Acids Res. 42(Database issue), D1063-D1069 (2014).

15. Oppenheim, A., Katzir, Y., Fibach, E., Goldfarb, A. \& Rachmilewitz, E. Hypomethylation of DNA derived from purified human erythroid cells correlates with gene activity of the beta-globin cluster. Blood 66, 1202-1207 (1985)

16. Motterlini, R. \& Otterbein, L. E. The therapeutic potential of carbon monoxide. Nat. Rev. Drug Discov. 9, 728-743 (2010).

17. Afshinnekoo, E. et al. Fundamental biological features of spaceflight: advancing the field to enable deep-space exploration. Cell 183, 1162-1184 (2020).

18. Stepanek, J., Blue, R. S. \& Parazynski, S. Space medicine in the era of civilian spaceflight. N. Engl. J. Med. 380, 1053-1060 (2019).

19. Klein, M. et al. Absence of neocytolysis in humans returning from a 3-week high-altitude sojourn. Acta Physiologica. 232, e13647 (2021).

20. Gore, C. J. et al. Altitude training and haemoglobin mass from the optimised carbon monoxide rebreathing method determined by a meta-analysis. $\mathrm{Br}$. J. Sports Med. 47(Suppl. 1), i31-i39 (2013).

21. Ganz, T. Anemia of inflammation. N. Engl. J. Med. 381, 1148-1157 (2019).

22. Mitlyng, B. L., Singh, J. A., Furne, J. K., Ruddy, J. \& Levitt, M. D. Use of breath carbon monoxide measurements to assess erythrocyte survival in subjects with chronic diseases. Am. J. Hematol. 81, 432-438 (2006).

23. Guralnik, J. M., Eisenstaedt, R. S., Ferrucci, L., Klein, H. G. \& Woodman, R. C. Prevalence of anemia in persons 65 years and older in the United States: evidence for a high rate of unexplained anemia. Blood 104, 2263-2268 (2004).

Publisher's note Springer Nature remains neutral with regard to jurisdictional claims in published maps and institutional affiliations.

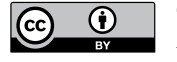

Open Access This article is licensed under a Creative Commons Attribution 4.0 International License, which permits use, sharing, adaptation, distribution and reproduction in any medium or format, as long as you give appropriate credit to the original author(s) and the source, provide a link to the Creative Commons license, and indicate if changes were made. The images or other third party material in this article are included in the article's Creative Commons license, unless indicated otherwise in a credit line to the material. If material is not included in the article's Creative Commons license and your intended use is not permitted by statutory regulation or exceeds the permitted use, you will need to obtain permission directly from the copyright holder. To view a copy of this license, visit http://creativecommons.org/licenses/by/4.0/.

(C) The Author(s) 2022 


\section{Methods}

Design and exposure. Between 2015 and 2020, astronauts were consecutively interviewed approximatively 1 year ahead of their ISS missions. The participating astronauts collected air and blood samples 3 months before Soyuz takeoff, four times onboard the ISS and serially after landing (total of 224 air and 196 blood samples; Fig. 1a,b). Each individual astronaut could have elected to participate in a complement of different experiments, some of which might have included additional venipunctures, causing blood and iron losses. To our knowledge, astronauts in this cohort were not screened for or confirmed to not be carrying any hemoglobinopathy or one of 1,000 known $\beta$-globin variants, some of which may affect hemoglobin levels in vivo (https://globin.bx.psu.edu/hbvar/) ${ }^{24,25}$. In this report, preflight measures served as the comparator to test the effects of exposure to space and determine whether one or more individual astronauts unknowingly had a $\beta$-globin variant (https://globin.bx.psu.edu/hbvar/).

Outcomes. The primary outcome was $\mathrm{CO}$ elimination quantified as the concentration of $\mathrm{CO}$ gas molecules [CO] in parts per billion eliminated through pulmonary ventilation. Increased $\mathrm{CO}$ elimination can arise from RBC destruction (intravascular or extravascular) in the spleen, liver or bone marrow. Clinically, whereas the first three conditions are called hemolysis, the latter is sometimes referred to as intramedullary hemolysis or ineffective erythropoiesis. In this paper, we defined, measured and discussed hemolysis as heme degradation products of the heme oxygenase enzymes arising from all sites. Secondary outcomes included other direct (iron) and indirect (bilirubin, transferrin percent saturation and ferritin) markers of heme degradation as well as haptoglobin, EPO, CBC and C-reactive protein ${ }^{26,27}$. CBC, reticulocytes and erythrocyte sedimentation rate (ESR) were not measured during flight, as they require analyses on fresh whole blood. In addition, measuring $\mathrm{CBC}$ and reticulocytes requires a hemocytometer, and measuring ESR requires gravity, both of which are unavailable on the ISS. In this trial, we did not measure the age of erythrocytes to comment on the subpopulation of RBCs present in space.

Laboratory analyses. Various markers of increased erythrocyte destruction intravascular or extravascular are used clinically, including the presence of schistocytes on a blood smear, free hemoglobin in blood, decreased haptoglobin for its high affinity with free hemoglobin, increased bilirubin (indirect or direct), increased ferritin, increased LDH, d-dimer and hemosideruria ${ }^{28}$. However, for different reasons, these markers are not precise, quantitative or direct markers of hemolysis ${ }^{28}$. Iron and transferrin saturation may rise with many types of anemia, including hypoproliferation/hypoplasia when iron is not cleared from the plasma. Similarly, serum ferritin can increase with any cause of anemia other than iron deficiency and/or bleeding. The iron in circulating RBCs is transferred to reticuloendothelial stores, which release a proportional amount of ferritin. For these reasons, we chose endogenous CO elimination as a precise, direct and quantitative marker of hemolysis as the primary outcome measure of the study. We measured CO elimination following the methods of Shahin et al. ${ }^{7}$. Briefly, upon waking up, after a 20 -s breath hold, astronauts exhaled through a mouthpiece connected to a one-way valve sequentially discarding $400 \mathrm{ml}$ of air from airways and filling a 750-ml collection bag with alveolar air (Supplementary Video 1). The alveolar air was immediately transferred to a vacuumed 200 -ml canister. Concurrently, the astronauts filled a second canister with ambient air. Canisters sampled on the ISS were stowed and boarded the next Earth-bound reentry vehicle. These methods accommodated for changes in pressure, temperature and vibration and maintained stable $[\mathrm{CO}]$ for at least 11 months ${ }^{7}$. Both air samples were extracted from the canisters and run through a gas chromatograph with a reduction gas detector. Subtracting the ambient air [CO] from the alveolar air [CO] provided the $\mathrm{CO}$ elimination.

Methods for secondary outcomes. The astronauts serially drew blood in tubes with double-polymer gel (serum separator tube) (Fig. 1). Blood was centrifuged at $1,515 \mathrm{~g}$ for $10 \mathrm{~min}$, and the tubes were frozen at $-80^{\circ} \mathrm{C}$. For reentry, they were loaded into the $-80^{\circ} \mathrm{C}$ freezer aboard the Dragon capsule and processed using commercial clinical analyzers for iron, transferrin percent saturation, ferritin, haptoglobin, bilirubin, EPO and C-reactive protein (Supplementary Table 5). Blood was also drawn pre- and postflight in EDTA tubes for CBC and reticulocytes at Johnson Space Center (JSC) clinical laboratory.

Blinding and anonymization. Each participant was assigned a random study number. The gas chromatograph operator was blinded to the subject identity and sampling time but, for calibration purposes, knew whether samples were ambient or alveolar. Aggregate data are presented instead of specific age, date or flight duration to prevent attributability.

Standard of care and ethics. Ethics approval was obtained from the National Aeronautics and Space Administration (NASA) Human Research Multilateral Review Board (\#Pro1283) and the Ottawa Health Sciences Research Ethics Board (\#2009646-01H). The trial was registered at NASA's Life Sciences Data Archives (https://lsda.jsc.nasa.gov/Experiment/exper/13399). All participants signed an informed consent form.
Assumptions and control for confounding. Preflight measures ensured that astronauts had no baseline anemia or hemolysis. Ambient [CO] varies widely. Given the higher and fluctuating ambient [CO] on the ISS, determining hemolysis based only on alveolar [CO] would have grossly overestimated $\mathrm{CO}$ elimination in space (Fig. 1c). In this trial, ambient air samples in the immediate environment of the astronaut were collected with each alveolar air sample. While altered pulmonary ventilation in space constitutes a potential unmeasured confounder, previous experiments reported uniform ventilation and similar ventilation/ perfusion ratio in space as on Earth ${ }^{29}$. Disrupted circadian cycle in orbit (16 sunrises and sunsets per $24 \mathrm{~h}$ ) may affect hemolysis ${ }^{30}$. We controlled for this potential confounder by chronostandardizing the sampling according to the astronaut schedule set on Greenwich meridian time. Nonhemoglobin heme proteins (cytochromes and myoglobin) are catalyzed by heme oxygenases to generate $15 \%$ of the human $\mathrm{CO}^{6}$, but this has never been measured in space. No liver or muscle necrosis was reported in space ${ }^{31}$, and muscle atrophy in space was addressed through exercise countermeasures. Earth-based data on muscle atrophy showed negligible changes in muscle myoglobin content ${ }^{32,33}$. Finally, myoglobin content is approximately eightfold less than hemoglobin content, decreasing the likelihood that nonhemoglobin heme degradation biased the interpretation of $\mathrm{CO}$ elimination data ${ }^{32}$. Entering space triggers a $10-12 \%$ blood volume contraction due to fluid shifts, which leads to hemoconcentration ${ }^{1,2,34}$. We did not apply a $10-12 \%$ correction to the initial ISS data, because serum analytes have short half-lives. In addition, Haldane's equation determining $\mathrm{O}_{2}$ and $\mathrm{CO}$ binding to hemoglobin is unaffected by hemoconcentration. Importantly, ISS ambient pressure reproduces atmospheric pressure, validating Henry's law governing CO elimination according to partial pressure gradients.

Statistical analyses. For this exposure study, summary statistics are limited to point estimates with unadjusted $95 \% \mathrm{CI}$ widths. No $P$ values are provided. We also present mean percent increases from baseline. Because a few samples were damaged, the ratio of estimates at individual time points may differ slightly from the percent increase estimates based on paired observations. Paired observations were necessary to calculate CIs on percent increase.

Reporting Summary. Further information on research design is available in the Nature Research Reporting Summary linked to this article.

\section{Data availability}

Aggregated data to understand and assess the conclusions of this research are available in the figures and supplementary tables. Individual astronaut source data have been deposited in NASA's Life Sciences Data Archives. Investigators can request access to the astronaut data at https://lsda.jsc.nasa.gov/.

\section{References}

24. Borg, J. et al. Haploinsufficiency for the erythroid transcription factor KLF1 causes hereditary persistence of fetal hemoglobin. Nat. Genet. 42, 801-805 (2010).

25. Borg, J., Patrinos, G. P., Felice, A. E. \& Philipsen, S. Erythroid phenotypes associated with KLF1 mutations. Haematologica 96, 635-638 (2011).

26. Gunga, H. C. et al. Erythropoietin under real and simulated microgravity conditions in humans. J. Appl. Physiol. 81, 761-773 (1996).

27. Gunga, H. C., Kirsch, K., Roecker, L. \& Jelkmann, W. Haemopoietic, thrombopoietic, and vascular endothelial growth factor in space. Lancet 353, 470 (1999).

28. Barcellini, W. \& Fattizzo, B. Clinical applications of hemolytic markers in the differential diagnosis and management of hemolytic anemia. Dis. Markers 2015, 635670 (2015)

29. Shelhamer, M. et al. Selected discoveries from human research in space that are relevant to human health on Earth. NPJ Microgravity 6, 5 (2020).

30. Panda, S. Circadian physiology of metabolism. Science 354, 1008-1015 (2016).

31. Beheshti, A. et al. Multi-omics analysis of multiple missions to space reveal a theme of lipid dysregulation in mouse liver. Sci. Rep. 9, 19195 (2019).

32. Jansson, E., Sylven, C., Arvidsson, I. \& Eriksson, E. Increase in myoglobin content and decrease in oxidative enzyme activities by leg muscle immobilization in man. Acta Physiol. Scand. 132, 515-517 (1988).

33. Lee, J. U. et al. Increase of myoglobin in rat gastrocnemius muscles with immobilization-induced atrophy. J. Phys. Ther. Sci. 25, 1617-1620 (2013).

34. Fischer, C. L., Johnson, P. C. \& Berry, C. A. Red blood cell mass and plasma volume changes in manned space flight. JAMA 200, 579-583 (1967).

\section{Acknowledgements}

We thank all 14 participants, who generously consented to the collection of these specimens in addition to their other mission duties, as well as the NASA Increment Science Coordinators at JSC and Reta Warren at the JSC Clinical Laboratory for the care of the blood samples. Special thanks to Steven Beck of the JSC Toxicology and Environmental Chemistry laboratory and his team for conditioning the canisters used for this study and to Doug Worthy and Michele Rauh of the Green House Gases Program at Environment Canada for their assistance with the gas chromatograph methods and 
dilution testing of the $\mathrm{CO}$ controls used in this study; Sandy Bookalam and her team at the Eastern Ontario Regional Laboratory Association and Cathy Andrusiak at In-Common Laboratories for blood analyses; Theresa Backlund, the study coordinator for MARROW; Nicole Buckley, Didier Yovo, Denis Charlebois, Stanislav Chrastina, Bruce Nicayenzi and Valerie Gill from Space Exploration at the Canadian Space Agency; Carolynn Cook for preparing the graphs; Martin Richard for video production; and Hans Uhthoff for his critical review of the manuscript. This work was funded by Canadian Space Agency Contracts 9F053-120589 to G.T. and 9F008-140254 to G.T., O.L. and H.L. The funder was not involved in the analysis, decision to publish or preparation of the manuscript.

\section{Author contributions}

G.T. was responsible for the conception and direction of the study. G.T. and H.L. established methods and supervised measures. G.T., N.S. and T.R. were responsible for data treatment and analysis. G.T., H.L., N.S., O.L. and T.R. contributed to data interpretation and manuscript writing.

\section{Competing interests}

The authors declare no competing interests.

\section{Additional information}

Supplementary information The online version contains supplementary material available at https://doi.org/10.1038/s41591-021-01637-7.

Correspondence and requests for materials should be addressed to Guy Trudel.

Peer review information Nature Medicine thanks Lawrence Rice, Hanns-Christian Gunga, Joseph Borg and the other, anonymous, reviewer(s) for their contribution to the peer review of this work. Michael Basson was the primary editor on this article and managed its editorial process and peer review in collaboration with the rest of the editorial team.

Reprints and permissions information is available at www.nature.com/reprints. 


\section{natureresearch}

Corresponding author(s):

Guy Trudel

Last updated by author(s): 2021/11/12

\section{Reporting Summary}

Nature Research wishes to improve the reproducibility of the work that we publish. This form provides structure for consistency and transparency in reporting. For further information on Nature Research policies, see Authors \& Referees and the Editorial Policy Checklist.

\section{Statistics}

For all statistical analyses, confirm that the following items are present in the figure legend, table legend, main text, or Methods section.

$\mathrm{n} / \mathrm{a}$ Confirmed

$\bigotimes$ The exact sample size $(n)$ for each experimental group/condition, given as a discrete number and unit of measurement

$\square$ \ A statement on whether measurements were taken from distinct samples or whether the same sample was measured repeatedly

$\triangle \square$ The statistical test(s) used AND whether they are one- or two-sided

$\triangle \square$ Only common tests should be described solely by name; describe more complex techniques in the Methods section.

$\bigotimes$ A description of all covariates tested

Х $\square$ A description of any assumptions or corrections, such as tests of normality and adjustment for multiple comparisons

A full description of the statistical parameters including central tendency (e.g. means) or other basic estimates (e.g. regression coefficient) AND variation (e.g. standard deviation) or associated estimates of uncertainty (e.g. confidence intervals)

$\square$ For null hypothesis testing, the test statistic (e.g. $F, t, r$ ) with confidence intervals, effect sizes, degrees of freedom and $P$ value noted

Wive $P$ values as exact values whenever suitable.

Х For Bayesian analysis, information on the choice of priors and Markov chain Monte Carlo settings

Х $\square$ For hierarchical and complex designs, identification of the appropriate level for tests and full reporting of outcomes

Х $\square$ Estimates of effect sizes (e.g. Cohen's $d$, Pearson's $r$ ), indicating how they were calculated

Our web collection on statistics for biologists contains articles on many of the points above.

\section{Software and code}

Policy information about availability of computer code

Data collection No software was used for data collection

Data analysis Data analysis was carried out using Microsoft Excel Version 2102.

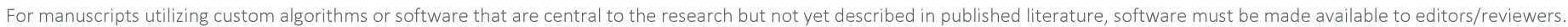
We strongly encourage code deposition in a community repository (e.g. GitHub). See the Nature Research guidelines for submitting code \& software for further information.

\section{Data}

Policy information about availability of data

All manuscripts must include a data availability statement. This statement should provide the following information, where applicable:

- Accession codes, unique identifiers, or web links for publicly available datasets

- A list of figures that have associated raw data

- A description of any restrictions on data availability

Aggregated data to understand and assess the conclusions of this research are available in the Figures and Supplementary Tables. Astronaut source data are restricted for confidentiality. Individual astronaut data are freely available by request to the NASA's Life Sciences Data Archives (LSDA) at https://Isda.jsc.nasa.gov/ Access to human data will be granted following a review and JSC IRB approval. 
Please select the one below that is the best fit for your research. If you are not sure, read the appropriate sections before making your selection.

Х Life sciences

Behavioural \& social sciences

Ecological, evolutionary \& environmental sciences

For a reference copy of the document with all sections, see nature.com/documents/nr-reporting-summary-flat.pdf

\section{Life sciences study design}

All studies must disclose on these points even when the disclosure is negative.

Sample size 20 astronauts were presented with the MARROW protocol. 15 astronauts agreed to participate. 1 astronaut was not provided a mission during the duration of MARROW study. 11 male and 3 female astronauts completed the trial. Considering a baseline endogenous $\mathrm{CO}$ of $1600 \mathrm{ppb}$ with sd of 400ppb, 14 astronauts allowed detecting a 300ppb difference between Earth and space CO at an alpha level of 0.05 with $80 \%$ power (https://clincalc.com/stats/samplesize.aspx)

Data exclusions 12 air samples were excluded due collection, transfer or logistical errors. 5 blood samples in serum separation tubes (SST) were damaged at re-entry and yielded invalid result for one or more analytes. 1 EDTA tube for CBC was not collected. Therefore 212 measures of CO, 121 to 125 measures of specific serum analytes and 69 measures of $C B C$ populated the dataset.

Replication The air samples were measured for CO concentration in quintiplicates or until signal stabilization as per Shahin, N., Louati, H. \& Trudel, G. Measuring Human Hemolysis Clinically and in Extreme Environments Using Endogenous Carbon Monoxide Elimination. Ann Biomed Eng. 48, 1540-50 (2020). The blood samples were not sufficiently large to run duplicates.

Randomization Randomization is not applicable, Astronauts recruited to the MARROW protocol performed serial measures at prespecified time points preflight, onboard the International Space Station (ISS) and postflight up to 1 year after their space missions.

Blinding Each participant was assigned a random study number. The gas chromatograph operator was blinded to the subject identity and sampling time but, for calibration purposes, knew whether samples were ambient or alveolar. Aggregate data are presented instead of specific age, date or flight duration to prevent attributability.

\section{Behavioural \& social sciences study design}

All studies must disclose on these points even when the disclosure is negative.

Study description

Research sample

Sampling strategy

Data collection

Timing

Data exclusions

Non-participation

Randomization
Briefly describe the study type including whether data are quantitative, qualitative, or mixed-methods (e.g. qualitative cross-sectional, quantitative experimental, mixed-methods case study).

State the research sample (e.g. Harvard university undergraduates, villagers in rural India) and provide relevant demographic information (e.g. age, sex) and indicate whether the sample is representative. Provide a rationale for the study sample chosen. For studies involving existing datasets, please describe the dataset and source.

Describe the sampling procedure (e.g. random, snowball, stratified, convenience). Describe the statistical methods that were used to predetermine sample size $O R$ if no sample-size calculation was performed, describe how sample sizes were chosen and provide a rationale for why these sample sizes are sufficient. For qualitative data, please indicate whether data saturation was considered, and what criteria were used to decide that no further sampling was needed.

Provide details about the data collection procedure, including the instruments or devices used to record the data (e.g. pen and paper, computer, eye tracker, video or audio equipment) whether anyone was present besides the participant(s) and the researcher, and whether the researcher was blind to experimental condition and/or the study hypothesis during data collection.

Indicate the start and stop dates of data collection. If there is a gap between collection periods, state the dates for each sample cohort.

If no data were excluded from the analyses, state so OR if data were excluded, provide the exact number of exclusions and the rationale behind them, indicating whether exclusion criteria were pre-established.

State how many participants dropped out/declined participation and the reason(s) given OR provide response rate OR state that no participants dropped out/declined participation.

If participants were not allocated into experimental groups, state so OR describe how participants were allocated to groups, and if allocation was not random, describe how covariates were controlled. 


\section{Ecological, evolutionary \& environmental sciences study design}

All studies must disclose on these points even when the disclosure is negative.

$\begin{array}{ll}\text { Study description } & \begin{array}{l}\text { Briefly describe the study. For quantitative data include treatment factors and interactions, design structure (e.g. factorial, nested, } \\ \text { hierarchical), nature and number of experimental units and replicates. }\end{array}\end{array}$

Research sample Describe the research sample (e.g. a group of tagged Passer domesticus, all Stenocereus thurberi within Organ Pipe Cactus National Monument), and provide a rationale for the sample choice. When relevant, describe the organism taxa, source, sex, age range and any manipulations. State what population the sample is meant to represent when applicable. For studies involving existing datasets, describe the data and its source.

Sampling strategy

Note the sampling procedure. Describe the statistical methods that were used to predetermine sample size OR if no sample-size calculation was performed, describe how sample sizes were chosen and provide a rationale for why these sample sizes are sufficient.

Data collection

Describe the data collection procedure, including who recorded the data and how.

Timing and spatial scale

Indicate the start and stop dates of data collection, noting the frequency and periodicity of sampling and providing a rationale for these choices. If there is a gap between collection periods, state the dates for each sample cohort. Specify the spatial scale from which the data are taken

Data exclusions

If no data were excluded from the analyses, state so OR if data were excluded, describe the exclusions and the rationale behind them, indicating whether exclusion criteria were pre-established.

Reproducibility

Describe the measures taken to verify the reproducibility of experimental findings. For each experiment, note whether any attempts to repeat the experiment failed OR state that all attempts to repeat the experiment were successful.

Randomization

Describe how samples/organisms/participants were allocated into groups. If allocation was not random, describe how covariates were controlled. If this is not relevant to your study, explain why.

Blinding

Describe the extent of blinding used during data acquisition and analysis. If blinding was not possible, describe why OR explain why blinding was not relevant to your study.

Did the study involve field work? $\square$ Yes $\square$ No

Field work, collection and transport

Field conditions

Location

Access and import/export

Disturbance
Describe the study conditions for field work, providing relevant parameters (e.g. temperature, rainfall).

State the location of the sampling or experiment, providing relevant parameters (e.g. latitude and longitude, elevation, water depth).

Describe the efforts you have made to access habitats and to collect and import/export your samples in a responsible manner and in compliance with local, national and international laws, noting any permits that were obtained (give the name of the issuing authority, the date of issue, and any identifying information).

\section{Reporting for specific materials, systems and methods}

We require information from authors about some types of materials, experimental systems and methods used in many studies. Here, indicate whether each material, system or method listed is relevant to your study. If you are not sure if a list item applies to your research, read the appropriate section before selecting a response.

\begin{tabular}{l|l} 
Materials \& experimental sys \\
\hline $\mathrm{n} / \mathrm{a}$ & Involved in the study \\
$\searrow$ & $\square$ Antibodies \\
$\searrow$ & $\square$ Eukaryotic cell lines \\
$\square$ & $\square$ Palaeontology \\
$\searrow$ & $\square$ Animals and other organisms \\
$\square$ & $\bigotimes$ Human research participants \\
$\square$ & $\bigotimes$ Clinical data
\end{tabular}

Methods $\mathrm{n} / \mathrm{a}$ Involved in the study

Х $\square$ ChIP-seq

Х $\square$ Flow cytometry

Х $\square$ MRI-based neuroimaging 
Antibodies used

Validation
Describe all antibodies used in the study; as applicable, provide supplier name, catalog number, clone name, and lot number Describe the validation of each primary antibody for the species and application, noting any validation statements on the manufacturer's website, relevant citations, antibody profiles in online databases, or data provided in the manuscript.

\section{Eukaryotic cell lines}

Policy information about cell lines

Cell line source(s)

State the source of each cell line used.

Authentication

Describe the authentication procedures for each cell line used OR declare that none of the cell lines used were authenticated.

Mycoplasma contamination

Confirm that all cell lines tested negative for mycoplasma contamination OR describe the results of the testing for mycoplasma contamination OR declare that the cell lines were not tested for mycoplasma contamination.

Commonly misidentified lines

(See ICLAC register)

Name any commonly misidentified cell lines used in the study and provide a rationale for their use.

\section{Palaeontology}

Specimen provenance

Provide provenance information for specimens and describe permits that were obtained for the work (including the name of the issuing authority, the date of issue, and any identifying information).

Specimen deposition

Indicate where the specimens have been deposited to permit free access by other researchers.

Dating methods

If new dates are provided, describe how they were obtained (e.g. collection, storage, sample pretreatment and measurement), where they were obtained (i.e. lab name), the calibration program and the protocol for quality assurance OR state that no new dates are provided.

Tick this box to confirm that the raw and calibrated dates are available in the paper or in Supplementary Information.

\section{Animals and other organisms}

Policy information about studies involving animals; ARRIVE guidelines recommended for reporting animal research
Laboratory animals
For laboratory animals, report species, strain, sex and age OR state that the study did not involve laboratory animals.
Wild animals
Provide details on animals observed in or captured in the field; report species, sex and age where possible. Describe how animals were caught and transported and what happened to captive animals after the study (if killed, explain why and describe method; if released, say where and when) OR state that the study did not involve wild animals.

Field-collected samples

For laboratory work with field-collected samples, describe all relevant parameters such as housing, maintenance, temperature, photoperiod and end-of-experiment protocol OR state that the study did not involve samples collected from the field.

Ethics oversight

Identify the organization(s) that approved or provided guidance on the study protocol, OR state that no ethical approval or guidance was required and explain why not.

Note that full information on the approval of the study protocol must also be provided in the manuscript.

\section{Human research participants}

Policy information about studies involving human research participants

Population characteristics

Recruitment

Ethics oversight
All astronauts participated in a planned 6 month excursion on board the International Space Station. 14 astronauts were recruited (11 men and 3 women; $45 \pm 7$ years)

Between 2015 and 2020, astronauts were consecutively interviewed approximatively 1 year ahead of their ISS missions. 20 astronauts were presented with the MARROW protocol. 15 astronauts agreed to participate. 1 astronaut was not provided a mission during the duration of MARROW study. There are fewer woman than men in the astronaut corps. Our recruitment was representative with more male astronauts recruited.

The protocol was approved by both the NASA JSC Human Research Multilateral Review Board \#Pro1283 and the Ottawa Health Sciences Research Ethics Board \#2009646-01H

Note that full information on the approval of the study protocol must also be provided in the manuscript. 
Policy information about clinical studies

All manuscripts should comply with the ICMJE guidelines for publication of clinical research and a completed CONSORT checklist must be included with all submissions.

Clinical trial registration

Study protocol

Data collection

Outcomes

The trial was registered at NASA's Life Sciences Data Archives https://Isda.jsc.nasa.gov/Experiment/exper/13399.
The study protocol will be uploaded through the manuscript submission portal.
The participating astronauts collected air and blood samples 3 months before Soyuz take off, 4 times onboard the ISS and serially
after landing
The primary outcome was CO elimination quantified as the concentration of CO gas molecules [CO] in parts per billion (ppb)
eliminated through pulmonary ventilation. Secondary outcomes included serum iron, bilirubin, transferrin \% saturation, ferritin,
haptoglobin, erythropoietin, C-reactive protein and complete blood count and were processed using commercial clinical
analyzers (Supplementary Table S5).

\section{ChIP-seq}

\section{Data deposition}

$\square$ Confirm that both raw and final processed data have been deposited in a public database such as GEO.

$\square$ Confirm that you have deposited or provided access to graph files (e.g. BED files) for the called peaks.

\section{Data access links}

May remain private before publication.

Files in database submission

Genome browser session

(e.g. $\underline{\operatorname{UCSC}}$ )

\section{Methodology}

Replicates

Sequencing depth

Antibodies

Peak calling parameters

Data quality

Software
For "Initial submission" or "Revised version" documents, provide reviewer access links. For your "Final submission" document, provide a link to the deposited data.

Provide a list of all files available in the database submission.

Provide a link to an anonymized genome browser session for "Initial submission" and "Revised version" documents only, to enable peer review. Write "no longer applicable" for "Final submission" documents.
Describe the experimental replicates, specifying number, type and replicate agreement.

Describe the sequencing depth for each experiment, providing the total number of reads, uniquely mapped reads, length of reads and whether they were paired-or single-end.

Describe the antibodies used for the ChIP-seq experiments; as applicable, provide supplier name, catalog number, clone name, and lot number.

Specify the command line program and parameters used for read mapping and peak calling, including the ChIP, control and index files used.

Describe the methods used to ensure data quality in full detail, including how many peaks are at FDR 5\% and above 5-fold enrichment.

Describe the software used to collect and analyze the ChIP-seq data. For custom code that has been deposited into a community repository, provide accession details.

\section{Flow Cytometry}

\section{Plots}

Confirm that:

$\square$ The axis labels state the marker and fluorochrome used (e.g. CD4-FITC).

$\square$ The axis scales are clearly visible. Include numbers along axes only for bottom left plot of group (a 'group' is an analysis of identical markers).

$\square$ All plots are contour plots with outliers or pseudocolor plots.

$\square$ A numerical value for number of cells or percentage (with statistics) is provided.

\section{Methodology}

Sample preparation

Instrument
Describe the sample preparation, detailing the biological source of the cells and any tissue processing steps used.

Identify the instrument used for data collection, specifying make and model number. 
Software

Cell population abundance

Gating strategy
Describe the software used to collect and analyze the flow cytometry data. For custom code that has been deposited into a community repository, provide accession details.

Describe the abundance of the relevant cell populations within post-sort fractions, providing details on the purity of the samples and how it was determined.

Describe the gating strategy used for all relevant experiments, specifying the preliminary FSC/SSC gates of the starting cell population, indicating where boundaries between "positive" and "negative" staining cell populations are defined.

Tick this box to confirm that a figure exemplifying the gating strategy is provided in the Supplementary Information.

Magnetic resonance imaging

Experimental design

Design type

Design specifications

Behavioral performance measures

Acquisition

Imaging type(s)

Field strength

Sequence \& imaging parameters

Area of acquisition

Diffusion MRI

Used

\section{Preprocessing}

\section{Preprocessing software}

Normalization

Normalization template

Noise and artifact removal

Volume censoring

Statistical modeling \& inference

Model type and settings

Effect(s) tested

Specify type of analysis: Whole brain

Provide detail on software version and revisior
segmentation, smoothing kernel size, etc.).

If data were normalized/standardized, describe the approach(es): specify linear or non-linear and define image types used for transformation $O R$ indicate that data were not normalized and explain rationale for lack of normalization.

Describe the template used for normalization/transformation, specifying subject space or group standardized space (e.g. original Talairach, MNI305, ICBM152) OR indicate that the data were not normalized.

Describe your procedure(s) for artifact and structured noise removal, specifying motion parameters, tissue signals and physiological signals (heart rate, respiration).

Define your software and/or method and criteria for volume censoring, and state the extent of such censoring.

Specify type (mass univariate, multivariate, RSA, predictive, etc.) and describe essential details of the model at the first
Statistic type for inference (See Eklund et al. 2016)

Correction ANOVA or factorial designs were used. and second levels (e.g. fixed, random or mixed effects; drift or auto-correlation).

Define precise effect in terms of the task or stimulus conditions instead of psychological concepts and indicate whether brain $\square$ ROl-based $\square$ Both

Specify voxel-wise or cluster-wise and report all relevant parameters for cluster-wise methods.

Describe the type of correction and how it is obtained for multiple comparisons (e.g. FWE, FDR, permutation or Monte (carlo) 
Models \& analysis

$\mathrm{n} / \mathrm{a} \mid$ Involved in the study

$\square \square$ Functional and/or effective connectivity

$\square \square$ Graph analysis

$\square$ Multivariate modeling or predictive analysis

Functional and/or effective connectivity

Graph analysis

Multivariate modeling and predictive analysis
Report the measures of dependence used and the model details (e.g. Pearson correlation, partial correlation, mutual information).

Report the dependent variable and connectivity measure, specifying weighted graph or binarized graph, subject-or group-level, and the global and/or node summaries used (e.g. clustering coefficient, efficiency, etc.).

Specify independent variables, features extraction and dimension reduction, model, training and evaluation metrics. 